\title{
Monitorização seqüencial do transplante renal com citologia aspirativa
}

\author{
R.C. MAnfRo, L.F.S. GonçALVES, L.A.R DE MOURA \\ Unidade de Transplante Renal - Serviço de N efrologia - Hospital de Clínicas de Porto Alegre, Universidade F ederal do Rio Grande do \\ Sul, Porto Alegre, RS.
}

RESUMO - Oвj etivo. Avaliar a utilidade da citologia aspirativa renal convencional na monitorização seqüencial da rejeição aguda de transplantes renais.

Material e Método. 376 punções aspirativas renais em 30 pacientes transplantados. Os diagnósticos das situações clínicas em que as aspirações foram feitas foram estabelecidos de maneira independente.

Resultados. Na avaliação seqüencial "cega" obteve-se $82,7 \%$ de representatividade das amostras. Encontraram-se aumentos significativos do incremento corrigido total (ICT) e dos números de células imunoativadas por lâmina nos episódios de rejeição aguda quando comparados aos valores obtidos durante os períodos de função estável do enxerto, necrose tubular aguda e nefrotoxicidade por ciclosporina. Os parâmetros diagnósticos para rejeição aguda foram: sensibilidade: $71,8 \%$; espe-

\section{INTRODUÇÃO}

O transplante renal é, atualmente, a melhor opção terapêutica e de reabilitação para pacientes com insuficiência renal crônica terminal ${ }^{1-3}$. Apesar disso, esta é uma forma de tratamento que ainda se encontra longe da perfeição, uma vez que órgãos transplantados são perdidos por diversas causas.

As rejeições aguda e crônica são as principais causas de perda de rins transplantados e decorrem, principalmente, da falta de identidade genética entre doador e receptor, com bloqueio incompleto da resposta imune ${ }^{4,5}$. Como não existe, atualmente, terapêutica comprovadamente eficiente para a rejeição crônica, a prevenção, diagnóstico e tratamento da rejei ção aguda assumem importância primordial no manejo do paciente transplantado renal, pois a sua cura incompleta pode ser o mecanismo inicial perpetuador dos processos de rejeição crônica ${ }^{5,6}$.

$\mathrm{Na}$ prática clínica, o aumento da creatinina sérica é a principal pista para o diagnóstico de rejeição aguda. No entanto, este dado pode não estar presente, como nos casos de necrose tubular aguda (NTA), ou ocorrer em diversas outras cir- cificidade: $87,3 \%$; valor preditivo positivo: $50,9 \%$; valor preditivo negativo: $94,4 \%$; e acurácia: $84,9 \%$. Os resultados falsos-positivos para rejeição foram devidos, principalmente, a infecção citomegálica ou subseqüentes ao uso de OKT 3 para tratamento de episódios de rejeição aguda celular. E m 10 dos 11 resultados falsos-negativos, encontrou-se o diagnóstico de imunoativação incipiente, que deve ser considerado como um alerta para a possibilidade de rejeição aguda.

Conclusões. A citologia aspirativa renal é um método útil na monitorização seqüencial da rejeição aguda no paciente transplantado renal. Os melhores resultados são obtidos quando os dados da citologia aspirativa são interpretados juntamente com o quadro clínico.

UNITERMOS: Transplante renal - rejeição aguda. Citologia aspirativa.

cunstâncias, como nefrotoxicidade por ciclosporina, pielonefrite aguda, obstrução e/ou fístula urinária, uso de drogas nefrotóxicas ou, ainda, diminuição da perfusão renal por problemas vasculares. Apesar de diversos métodos de monitorização não invasiva terem sido tentados, permanece a biópsia renal o "padrão-ouro" para a avaliação das disfunções dos rins transplanta$\operatorname{dos}^{7,8}$. Contudo, a biópsia renal apresenta algumas limitações, como os riscos, os problemas de amostragem, a informação é estática - e os efeitos da terapia na morfologia são, na maior parte das vezes, presumidos, uma vez que o procedimento não deve ser repetido com muita freqüência ${ }^{9,10}$.

A punção aspirativa renal (PAR) foi, inicialmente, aplicada ao transplante renal em 1968, quando, em Helsinque, se utilizou esse procedimento em aloenxertos renais humanos ${ }^{11}$. Estudos subseqüentes serviram para a padronização técnica do procedimento e do método, sendo definidos parâmetros de representatividade da amostra e introduzida a metodologia de inter pretação conhecida como "incremento corrigido total" (ICT), que é uma tentativa de quantificar o infiltrado inflamatório intra-enxerto ${ }^{12,13}$. A reprodutibilidade da 
PAR, analisada através do método do ICT, foi avaliada por diferentes estratégi as e pode ser considerada satisfatória ${ }^{14,15}$. A avaliação do estado das células parenquimatosas, mais especificamente das cél ulas tubulares e endoteliais, é feita através de um escore morfológico.

Este trabalho foi realizado com o objetivo de avaliar a acuidade diagnóstica da PAR na monitorização seqüencial da rejeição aguda em pacientes transplantados renais.

\section{PACIENTES E MÉTODOS}

Foram incluídos 30 pacientes transplantados no período de novembro de 1990 a abril de 1992 na Unidade de Transplante Renal do Hospital de Clínicas de Porto Alegre. Todos os pacientes apresentavam prova cruzada negativa e iniciaram a medicação imunossupressora no período pré-transplante imediato (receptores de rins de doador cadáver) ou dois dias antes da cirurgia do transplante (receptores de rins de doador vivo). Dois pacientes estavam sendo submetidos a retransplante, 12 receberam rins de doador cadáver, 17 receberam rins de doadores parentes e um recebeu o rim do cônjuge.

A avaliação clínica e o acompanhamento laboratorial pós-transplante foram feitos diariamente durante a internação; duas vezes por semana por um mês após a alta hospitalar; uma vez por semana até o fim do segundo mês; quinzenal mente, por 4 meses adicionais e, mensalmente, daí por diante, até completar um ano. O acompanhamento laboratorial básico constou de exames bioquímicos e hematológicos. No pós-operatório imediato foi obtido, rotineiramente, um ultra-som e uma cintilografia de fluxo renal com ${ }^{99 m T C-D T P A . ~ D o s a g e n s ~ d e ~}$ ciclosporina (Abbott Laboratories, EUA), em sangue total utilizando anticorpo policlonal, foram obtidas duas vezes por semana durante a internação e por indicação clínica, posteriormente.

As disfunções agudas do enxerto, conceituadas como aumento confirmado e significativo $(>0,3 \mathrm{mg} /$ $\mathrm{dL}$ ) da creatinina sérica, ou ausência da queda aos níveis esperados no pós-operatório, acompanhados ou não de quadro clínico de rejeição aguda, foram investigadas com cintilografia de fluxo, ecografia, dosagem de cicl osporina sérica, exame do sedimento urinário e exame cultural da urina. A punção biópsia renal (PBR) foi realizada sempre que houvesse disfunção aguda do enxerto, não existisse contraindicação absoluta e fosse operacional.

As punções aspirativas renais foram feitas duas vezes por semana durante a internação, uma vez por semana até o fim do primeiro mês pós-transplante, duas vezes durante o segundo mês, uma vez no terceiro mês, e nos episódios de disfunção aguda do enxerto. Tanto as PAR quanto as PBR foram feitas com o consentimento informado do paciente após o esclarecimento do procedimento, seus riscos e potenciais benefícios.

As rejeições agudas foram diagnosticadas com base no aumento da creatinina sérica, acompanhado ou não de quadro clínico típico, levando-se em conta os exames citados na avaliação de disfunção renal aguda do enxerto. Um último critério diagnóstico foi a resposta ao tratamento da rejeição aguda. Os episódios de nefrotoxicidade por ciclosporina foram diagnosticados na presença de quadro clínico compatível associado ou não a nível sérico elevado, biópsia renal compatível ou, ainda, melhora da função renal com diminuição ou suspensão da droga. O diagnóstico de NTA foi de exclusão, tendo sido feito na ausência de rejeição, nefrotoxicidade, pielonefrite ou de outras condições conhecidas. Arbitrariamente, diagnosticouse como estando em NTA todo o paciente sem as condições acima citadas e cuja creatinina sérica fosse superior a $2,5 \mathrm{mg} \%$ no período pós-transplante imediato.

Todos os diagnósticos da situação clínica no dia da PAR foram estabelecidos de maneira independente usando-se os critéri os citados acima, sem, no entanto, utilizar as informações da citologia aspirativa na elaboração diagnóstica.

As PAR foram realizadas à beira do leito com localização do rim por pal pação. A pós anti-sepsia, introduz-se agulha de punção espinhal $25 G$ 31/2 na região cortical, acopl ando-se a seringa e iniciandose a aspiração com movi mentos repetidos de vai-evem e de rotação. O material aspirado é diluído em RPMI e processado por citocentrifugação. U ma gota de sangue periférico é processada da mesma maneira. Os citoesfregaços das PAR e do sangue são corados por MGG (May-Grünwald-Giemsa) e interpretados de acordo com o método do $\mathrm{ICT}^{13}$.

Para o diagnóstico citológico de rejeição aguda utilizou-se o escore do ICT maior ou igual a 3,0, acompanhado de "sinais de imunoativação" assim definidos: a) imunoativação: células imunoativadas (plamócitos, linfoblastos, ou linfócitos ativados) representando mais de $1 \%$ do número total de leucócitos presentes na lâmina, ou presença na lâmina de 5 ou mais células imunoativadas; b) imunoativação incipiente: células imunoativadas presentes mas inferiores a $1 \%$ do total de leucócitos, ou células imunoativadas $>1$ e $<4$ por lâmina; c) ausência de imunoativação: células imunoativadas ausentes na lâmina. A presença de imunoativação incipiente al ertava para a possibilidade de rejeição aguda sem, no entanto, confirmá-la. 
Os protocol os das fichas de leituras foram identificados por um número, e as punções de um mesmo paciente, col ocadas em ordem cronológica para avaliação seqüencial por um único citologista que, assim, foi tornado "cego" para a identidade do paciente, o tipo de imunossupressão e o quadro clínico.

As punções biópsias renais foram executadas através das técnicas habituais. As colorações utilizadas foram: hematoxilina e eosina, PAS, PASM (prata metenamina) e tricrômico verde. A interpretação das lâminas foi feita por um único patologista, que era conhecedor do quadro clínico mas não das hipóteses diagnósticas ou das impressões clínicas. Os critérios diagnósticos empregados para esta inter pretação foram os recomendados no estudo Banff ${ }^{16}$. O resultado da PBR e/ou a evolução do paciente com as diferentes intervenções para tratamento de rejeição aguda foram utilizados como "padrão-ouro" para o estabelecimento dos parâmetros de acuidade diagnóstica da PAR.

\section{Análises estatísticas}

F oram empregadas estatísticas descritivas para a caracterização da amostra e o teste de Kolmogorov-Smirnov para a avaliação da distribuição dos dados. Com a final idade de verificar diferenças entre dois ou mais grupos independentes, foram utilizados os testes de Mann-Whitney e KruskallWallis, respectivamente. Testes de comparações múltiplas (diferença mínima significativa) pertinentes foram efetuados. O nível de significância mínimo adotado foi o de $p<0,05^{17,18}$.

\section{RESULTADOS}

A sobrevida até o terceiro mês pós-transplante, momento até o qual foi feita a avaliação com citologia aspirativa, foi $86,7 \%$ e $100 \%$ para enxertos e pacientes, respectivamente.

A incidência de rejeição aguda foi de $76,6 \%$ nos primeiros três meses e em 12 meses. Vinte e três pacientes apresentaram 28 episódios de rejeição aguda nos primeiros três meses pós-transplante $(1,22 \pm 0,52$ episódios por paciente com rejeição). O anticorpo monoclonal anti-CD3 $\left(\right.$ OKT $\left.^{\circledR}\right)$ foi utilizado em seis oportunidades para o tratamento de rejeição aguda.

Os 30 pacientes foram submetidos a 376 PAR. A média de punções por paciente foi de $12,5 \pm 3,4$, com variação de 4 a 20 punções por paci ente. Oitenta e três por cento das punções coradas por MGG foram consideradas representativas, permitindo avaliação citológica. As causas de não-representatividade foram: a) punção hemorrágica (36 punções; $9,6 \%)$; b) punção com pouco material ou ausente
Tabela 1 - Incremento corrigido total. Resultados por situação clínica

\begin{tabular}{lrr}
\hline \multicolumn{1}{c}{ Diagnóstico clínico } & N & Média+DP \\
Função renal estável & 114 & $2,95 \pm 1,91$ \\
NTA & 74 & $2,63 \pm 2,03$ \\
Rejeição aguda & 39 & $4,46 \pm 1,47$ \\
RA em tratamento (esteróides) & 45 & $4,04 \pm 2,33$ \\
RA em tratamento (OKT3) & 16 & $3,19 \pm 1,85$ \\
Citomegalovírus & 10 & $4,94 \pm 2,96$ \\
Necrose cortical & 6 & $1,98 \pm 1,33$ \\
Nefrotoxicidade CyA & 4 & $0,65 \pm 0,41$ \\
Outros diagnósticos & 3 & $2,67 \pm 1,29$ \\
Total & 311 & \\
\hline
\end{tabular}

Kruskall-Wallis, Análise de variância não-paramétrica (ANOVA). $N=$ número de punções; $\mathrm{DP}=$ desvio padrão; NTA = necrose tubular aguda; $\mathrm{RA}=$ rejeição aguda; $\mathrm{CyA}=$ Ciclosporina. Qui-quadrado corrigido: 48,23 ; $\mathrm{p}<0,0001$.

("branca") (19 punções; 5,1\%); e punção não avali ável por problema técnico (9 punções; 2,4\%).

A análise da citologia aspirativa convencional foi feita com base nos dados obtidos na avaliação seqüencial dos casos, sendo aferidos O ICT e o número de células imunoativadas por lâmina. Para a análise do ICT, 311 resultados de citologias aspirativas renais foram analisados. As médias e os respectivos desvios padrões do ICT por situação clínica estão demonstrados na tabela 1.

A avaliação da tabela 1 demonstra que existem diferenças significativas nas distribuições dos resultados do ICT de acordo com a si tuação clínica em que ele foi calculado. $\mathrm{Na}$ análise de variância, as diferenças ocorreram de maneira significativa quando os valores dos ICT, nos casos de rejeição aguda sem tratamento, foram comparados aos obtidos durante períodos de função estável do enxerto, necrose tubular aguda, nefrotoxicidade por ci closporina e outros diagnósticos. Não ocorreram diferenças significativas nos valores do ICT quando o grupo de punções obtidas durante episódios de rejeição aguda foi comparado aos grupos com infecção por citomegal ovírus, rej eição aguda em tratamento com esteróides, rejeição aguda em tratamento com OKT3 e necrose cortical.

A contagem das células imunoativadas foi possível em 315 lâminas. As médias das contagens com os desvios padrões estão demonstradas na tabela 2. A avaliação desta tabela revela que existem diferenças significativas nas distribuições dos valores das células imunoativadas por lâmina nas diferentes situações clínicas. Os valores obtidos nos episódios de rejeição aguda, durante seus tratamentos, seja com corticosteróides ou com OKT3 e na infecção por citomegal ovírus, são os mais el eva- 
Tabela 2 - Número de células imunoativadas por lâmina. Resultados por situação clínica

\begin{tabular}{|lrr|}
\hline \multicolumn{1}{c}{ Diagnóstico clínico } & N & Média+DP \\
Função renal estável & 116 & $2,70 \pm 7,85$ \\
NTA & 75 & $2,80 \pm 7,73$ \\
Rejeição aguda & 39 & $11,46 \pm 13,56$ \\
RA em tratamento (esteróides) & 45 & $5,62 \pm 8,21$ \\
RA em tratamento (OKT3) & 17 & $7,00 \pm 7,78$ \\
Citomegalovírus & 10 & $10.40 \pm 12,10$ \\
Necrose cortical & 6 & $0,00-$ \\
Nefrotoxicidade CyA & 4 & $0,50 \pm 1,00$ \\
Outros diagnósticos & 3 & $8,23 \pm 11,26$ \\
Total & 315 & \\
\hline
\end{tabular}

Kruskall-Wallis, Análise de variância não-paramétrica. $\mathrm{N}=$ número de punções; DP = desvio padrão; NTA = necrose tubular aguda; RA = rejeição aguda; CyA = ciclosporina. Qui-quadrado corrigico: 84,94; $p$ $<0,0001$.

Tabela 3 - Avaliação dos parâmetros diagnósticos para rejeição aguda da citologia aspirativa convencional

\begin{tabular}{lllllll|}
\hline $\begin{array}{l}\text { Parâmetro } \\
\text { índice }\end{array}$ & $\begin{array}{c}\text { Ponto de } \\
\text { corte }\end{array}$ & Sens & Esp & VPP & VPN & Ac \\
ICT & $\geq 3$ & 87,2 & 60,8 & 29,1 & 96,3 & 64,9 \\
CIA & $\geq 5$ & 76,9 & 84,9 & 48,4 & 95,2 & 83,7 \\
ICT e CIA & 3 e 5 & 71,8 & 87,3 & 50,9 & 94,4 & 84,9 \\
\hline
\end{tabular}

dos. Observa-se uma tendência a diminuição no número de células imunoativadas por lâmina quando foram administradas altas doses de corticosteróides ou de OKT3. Diferenças estatisticamente significativas foram encontradas nas comparações entre os grupos com rejeição aguda, independente da presença de tratamento, e o grupo com função estável do enxerto. O valor obtido no grupo infecção por citomegalovírus não foi estatisticamente diferente do obtido no grupo com rejeição aguda.

$\mathrm{Na}$ avaliação dos parâmetros diagnósticos da citologia aspirativa foram excluídos os casos de rejeição aguda em tratamento com esteróides ou com OKT3. Na tabela 3 estão demonstrados os parâmetros diagnósticos do ICT e do número de células imunoativadas isoladamente ou em conjunto. A avaliação da tabela 3 revela que nessa amostra o parâmetro isolado de melhor acurácia diagnóstica e de melhor balanço entre sensibilidade e especificidade para rej eição aguda é a presença inequívoca de imunoativação (células imunoativadas por lâmina $>5$ ), seguida do ICT $>3$. A associação do ICT $>3$ e células imunoativadas $>5$ apresentou o mel hor desempenho na avaliação da acurácia diagnóstica. Mesmo assim, na presente amostra, obteve-se uma sensibilidade intermediária e baixo valor preditivo positivo.

$\mathrm{Na}$ avaliação do parâmetro de melhor desempenho no cálculo da acurácia, ou seja, ICT $>3,0$, e células imunoativadas por lâmina $>5$, encontraram-se 27 falsos-positivos e 11 falsos-negativos. Nas 27 PAR com diagnóstico falso-positivo para rejeição aguda, verificou-se que em 18 ocasiões havia imunoativação associada a infecção por citomegalovírus ou após o uso de OKT3; em duas ocasiões o falso-positivo precedeu, por 48 e 72 horas, um episódio de rejeição aguda clinicamente detectável e, em 7 oportunidades, não foi possível detectar uma potencial razão para o resultado falso-positivo. Em 10 das 11 PAR com diagnóstico falso-negativo para rejeição aguda havia imunoativação incipiente.

Vinte e um pacientes foram submetidos a 38 biópsias renais ( $1,3 \pm 1,3$ biópsias por pacientes; variação: 0 a 6 biópsias por paciente), sendo 35 $(92,1 \%)$ adequadas para o diagnóstico histopatológico. Duas biópsias foram realizadas em transoperatório de intercorrências; as demais foram obtidas por agulha conforme descrito anteriormente ${ }^{7}$. A avaliação da PAR nos casos com biópsia renal foi feita nas 30 ocasiões em que as PAR e PBR foram concomitantes e representativas. Entre esses, 23 punções apresentaram infiltrados intersticiais, interpretados como rejeição aguda na biópsia de fragmento. Em 13 o infiltrado não era acompanhado de componente de rejei ção vascular, em oito havia componente vascular mas com predomínio do componente celular, e nos dois restantes o componente vascular era mais importante. Excluindo-se as punções obti das durante epi sódios de rejeição aguda em tratamento com esteróides ou OKT3, restaram para a análise 23 pares de punções, quando, tomando-se como "padrão-ouro" o resultado da bi ópsia renal de fragmento, chegouse aos seguintes parâmetros diagnósticos da análise citológica para o diagnóstico de rejeição aguda: sensibilidade, 76,5\%; especificidade, $80,0 \%$; valor preditivo positivo, 92,9\%; valor preditivo negativo, $44,4 \%$, e acuidade, $73,9 \%$.

\section{DISCUSSÃo}

A análise de trabal hos recentes de grupos americanos, europeus e nacionais permite concluir que a representatividade média da PAR situa-se em torno de $80 \%{ }^{19-24}$. O método do ICT ${ }^{12}$ é o mais empregado para a quantificação do infiltrado inflamatório dos enxertos renais. Para contornar o problema 
da contaminação sanguínea do aspirado renal, é feita uma contagem diferencial do material obtido no rim e do material obtido por punção digital. O conteúdo de leucócitos encontrados no sangue é subtraído do encontrado na amostra renal e, quando existente, a maior quantidade de leucócitos provenientes do material renal é considerada como estando em excesso no rim. Com o objeti vo de enfatizar os diagnósticos de rejeição, o conceito do ICT foi introduzido em 1982. Em função de sua importância fundamental nos processos de rejeição celular aguda, as células blásticas das linhagens $T$ e $B$ receberam o fator de correção mais elevado.

Diversos trabalhos avaliaram a correlação da PAR com a BRP que vem sendo considerada como o "padrão-ouro" para a avaliação dos métodos diagnósticos em transplante renal. De uma maneira geral, pode-se afirmar que os resultados da citologia aspirativa guardam boa correlação com os da biópsia renal para o diagnóstico de rejeição agu$\mathrm{da}^{19,20,25,26}$, e que o ICT é diretamente proporcional à severidade da rejeição quando avaliada por histopatologia ${ }^{27-29}$. Adicionalmente, estudos empregando técnicas de imunoperoxidase em aspirados renais e em fragmentos de biópsia demonstraram existir boa correlação fenotípica na freqüência dos diferentes subtipos celulares nas duas amostras ${ }^{30}$.

Quando a avaliação e a interpretação dos resultados da citologia aspirativa são feitas à luz do quadro clínico, as informações sobre a presença ou ausência de disfunção renal e de outras condições clínicas freqüentes em pós-operatório de transplante renal podem ser de grande auxílio. Assim, na presente série, excetuando-se sete punções, os falsos-positivos ocorreram em períodos subseqüentes à administração de OKT3 ou em períodos de infecção por citomegal ovírus 27,31,32. As duas punções com diagnósticos "falsos-positivos" que precederam, por curto espaço de tempo, episódios de rejeição aguda foram assim consideradas devido a estratégia empregada de avaliar o resultado de cada PAR com a situação clínica estabelecida para aquele. Esses dois diagnósticos não poderiam de fato ser considerados falsos-positivos. Dez das 11 punções que levaram a diagnósticos falsos-negativos para rejeição aguda apresentavam imunoativação incipiente, o que no contexto clínico de disfunção do enxerto, para a qual outras causas tenham sido devidamente afastadas, poderiam ter contribuído para diagnosticar rejeição aguda. Assim, o rendimento diagnósti co da citologia aspirativa de transplantes renais é bastante superior quando os resultados são interpretados dentro do contexto clínico.
A variabilidade encontrada na literatura em relação aos parâmetros diagnósticos da PAR parece ser parcialmente devida a escol ha de diferentes pontos de corte no ICT empregados nos trabal hos. A maioria dos trabalhos usam pontos de corte entre 2,5 e 5,0. Além disso, alguns pesquisadores requerem apenas um determinado valor de ICT para fazer o diagnóstico de rejeição aguda, enquanto outros exigem a presença de imunoativação. Adicionalmente, a maneira como é feita a determinação da situação clínica, rejeição versus não-rejeição e a prevalência de rejeição na amostra podem modificar significativamente os valores preditivos $^{33}$. Esta, provavelmente, é a razão pela qual os parâmetros diagnósticos diferiram no subgrupo de pacientes com biópsia renal. Primeiro, houve nesse grupo uma el evada prevalência de rejeição aguda, suspeita pela qual a maior parte das biópsias foi efetivada, e, segundo, a avaliação histológica tende a diagnosticar rejeição aguda com maior freqüência em função do achado de infiltrados que podem ou não ter esse significado $16,34,35$. Esses fatores podem ser os responsáveis pelo baixo valor preditivo negativo encontrado no subgrupo de pacientes com biópsia de fragmento no presente trabalho e no de outros ${ }^{36}$. Por sua vez, a baixa acuidade da PAR nesse subgrupo de punções deveu-se ao baixo valor preditivo negativo encontrado.

Os valores dos ICT durante os epi sódios de rejeição aguda são, em geral, significativamente maiores do que os encontrados em outras situações clínicas ${ }^{21,36}$, exceto, possivelmente, nas fases iniciais da infecção por citomegal ovírus (CMV), quando a presença de linfócitos grandes granulares pode ser a responsável pelo aumento do incremento ${ }^{37,38}$. O aumento desses linfócitos pode ser mais importante no enxerto do que no sangue periférico, quando parece crescer uma a duas semanas após o seu aumento no rim, sugerindo, nesses casos, que o sítio primário da infecção pelo CMV possa ser o próprio enxerto 27,39 .

Os valores dos ICT foram maiores no grupo de punções obti das durante epi sódi os de rejeição aguda do que naqueles obtidos em outras situações, como função estável do enxerto, necrose tubular aguda e nefrotoxicidade por ciclosporina. Rejeição aguda, necrose tubular e nefrotoxicidade por ciclosporina são as situações clínicas que mais comumente causam disfunção do enxerto em pósoperatório recente de transplante renal, período no qual a PAR encontra a sua maior utilidade.

A infecção pelo citomegalovírus é uma importante causa de morbidade, perda de enxertos e mortalidade em pacientes transplantados renais. 
No presente trabalho, o diagnóstico de infecção por citomegalovírus foi feito com base no quadro clínico e no aumento de quatro vezes dos títulos de anticorpos anticitomegal ovírus em dois pacientes. Dez PAR foram efetuadas nestes dois pacientes durante o quadro de infecção caracterizado por febre, pneumonia e acometimento do trato gastrointestinal no primeiro, e de febre, leucopenia e alteração nas provas de função hepática no outro. Nenhum desses pacientes apresentou disfunção do enxerto durante ou logo após o quadro de infecção viral, e as médias dos ICT, assim como das células imunoativadas, não foram estatisticamente diferentes das encontradas nos episódios de rejeição aguda. E sses achados estão de acordo com os referi dos na literatura, em que se relatam incrementos elevados, sem manifestações clínicas ou laboratoriais outras de rejeição aguda ${ }^{27,38}$. Na infecção citomegálica, os achados citológicos contribuem para a menor especificidade e valor preditivo positivo da técnica da citologia aspirativa renal quando se avaliam os parâmetros diagnósticos para rejeição aguda. Na verdade, uma avaliação crítica dos trabalhos disponíveis na literatura em que se estudou a PAR para o diagnóstico de rejeição aguda de transplantes renais demonstra que os casos de infecção citomegálica foram, na maior parte das vezes, retirados da análise.

A avaliação do número de células imunoativadas na amostra tem sido sugeri da como um critério isolado de boa acurácia para o diagnóstico de rejeição aguda de enxertos renais ${ }^{22,40}$. Nesse trabal ho, encontrou-se que as células imunoativadas estão significativamente aumentadas nos episódios de rejeição aguda, que elas diminuem com o tratamento da rejeição e que, além da rejeição aguda, episódios de infecção, principalmente viral, estão associados a um aumento no seu número. Dados experimentais demonstram que o infiltrado blástico, ou seja, o aparecimento de células imunoativadas no enxerto, precede a expressão de antígenos HLA classe II na rejeição aguda, modificada ou não por imunossupressores, e que a imunossupressão retarda a expressão das moléculas HLA classe II, sem no entanto retardar a infiltração blástica ${ }^{41}$. E sses achados sugerem que o estímulo à expressão dos antígenos HLA-DR, bem como de moléculas de adesão, nas células do enxerto seja produzido por células do componente blástico do infiltrado através da liberação de fator(es) solúvel(eis). Essas estruturas representam "alvos" imunes ou aumentam a interação célula a célula no enxerto.

$\mathrm{Em}$ conclusão, encontrou-se que a PAR é um método útil na avaliação seqüencial de pacientes transplantados renais. Possivelmente, este método encontra sua maior utilidade nos casos que evoluem com NTA pós-transplante em que o diagnóstico da rejeição aguda é difícil. Adicionalmente, conforme demonstrado na literatura, a avaliação da expressão de antígenos HLA e de moléculas de adesão pode ser útil na elucidação de casos com escores limítrofes ${ }^{42,43}$.

\section{SUMMARY}

\section{Aspiration citology in the sequential monitoriza- tion of kidney allografts}

OвJ естіVE. To evaluate the utility of kidney aspiration cytology in the sequential monitorization of acute rejection in renal transplant patients.

Patients and Methods. Thirty patients were submitted to 376 aspirations. The clinical diagnoses were independently established.

RESULTS. The representativity of the samples reached $82.7 \%$. The total corrected increment index and the number of immunoactivated cells were higher during acute rejection as compared to normal allograft function, acute tubular necrosis, and cyclosporine nephrotoxicity. The parameters to the diagnosis of acute rejection were: sensitivity: $71.8 \%$, specificity: $87.3 \%$, positive predictive value: $50.9 \%$, negative predictive value: $94.9 \%$ and accuracy $84.9 \%$. The false positive results were mainly related to cytomegal ovirus infection or to the administration of OKT3. In 10 out of 11 false negative results incipient immunoactivation was present alerting to the possibility of acute rejection.

Conclusions. Kidney aspiration cytology is a useful tool for the sequential monitorization of acute rejection in renal transplant patients. The best results are reached when the results of aspiration cytology are analyzed with the clinical data. [Rev Ass Med Brasil 1998; 44(2): 87-93.]

KEY WORDS: Renal transplantation. Acute rejection. Aspiration cytology.

\section{REFERÊNCIAS BIBLIOGRÁFICAS}

1. Morris PJ . Results of Renal Transplantation. In Morris PJ ed. Kidney transplantation - Principles and practice. $4^{\text {th }}$ ed. Philadel phia, W.B. Saunders, 1994; 504-23

2. United States Renal Data System. Survival probabilities and causes of death. Am J Kidney Dis 1991; 18(suppl 2): 49-60.

3. Russell J D, Beecroft, ML, Ludwin D, Churchill DN. The quality of life in renal transplantation. A prospective study. Transplantation 1992; 54: 656-60.

4. Tilney NL, Kupiec-Weglinsky J W. The biology of acute transplant rejection. Ann Surg 1991; 214: 99-106.

5. Tilney NL, Whitney WD, Diamond J W, Kupiec-Weglinski J W, 
Adams DH. Chronic rejection - an undefined conundrum. Transplantation 1991; 52: 389-98.

6. Forbes RDC, Darden AG, Gomersali M, Guttman RD. Patterns of donor major histocompatibility complex antigen expression in rat heart grafts in a model of chronic vascular rejection. Transplant Proc 1989; 21: 447-8.

7. ManfroRC, LeeJ Y, Lewgoy J , Gonçalves LF, Edel weiss MI et al. O papel da biópsia renal percutânea no transplanterenal. Rev Ass Med Brasil 1994; 40: 108-12.

8. Matas AJ , Tellis VA, Sablay $L$ et al. The value of needle renal allograft biopsy. III. A prospective study. Surgery 1985; 98: 922-5.

9. Gault MH, Muehrcke RC. Renal biopsy: current views and controversies. Nephron 1983; 34: 1-34.

10. Wilczek HE. Percutaneous needle biopsy of renal allograft: A clinical safety evaluation of 1129 biopsies. Transplantation 1990; 50: 790-7.

11. Pasternack A. Fine-needle aspiration biopsy of human renal homografts. Lancet 1968; 2: 82-4.

12. Häyry $P$, von Willebrand $E$. Practical guidelines for fine needle aspiration biopsy of human renal allografts. Ann Clin Res (Helsinki) 1981; 13: 288-306.

13. Häyry $P$, von Willebrand $E$. Transplant aspiration cytology. Transplantation 1984; 38: 7-12.

14. von Willebrand $E, H$ äyry P. Reproductibility of fine needle aspiration biopsy. Analysis of 93 double biopsies. Transplantation 1984; 38: 314-6.

15. Manfro RC, Gonçalves LFS, Moura LAR. Reproductibility of fine-needle aspiration biopsy in the diagnosis of acute rejection of renal allografts. Nephrol Dial Transplant 1995; 10: 2.306-9.

16. Solez K, Axelsen RA, Benediktsson $\mathrm{H}$ et al. International standardization of criteria for the histologic diagnosis of renal allograft rejection: the Banff working classification of kidney transplant pathology. Kidney Int 1993; 44: 411-22.

17. Campos H. Análise de variância - Classificação simples. In Campos H (ed). Estatística experimental não paramétrica. 4a ed. São Paulo, Editora Universidade de São Paulo, 1983; 201-26.

18. Zar J H. Biostatistical analysis. $2^{\text {nd }}$ ed. New J ersey, PrenticeHall Inc. Englewood Cliffs, 1984.

19. Gray DWR, Richardson R, Hughes D et al. A prospective, randomized, blind comparison of three biopsy techniques in the management of patients after renal transplantation. Transplantation 1992; 53: 1.226-32.

20. Reinholt FP, Bohman SO, Wilczek H, von Willebrand E, Häyry $P$. Fine-needle aspiration cytology and conventional histology in 200 renal allografts. Transplantation 1990; 49: 910-2.

21. Danovich G, Nast CC, Wilkinson A, Rosenthal T. Evaluation of fine-needle aspiration biopsy in the diagnosis of renal transplant dysfunction. Am J Kidney Dis 1991; 17: 206-10.

22. Helderman J H, Hernandez J, Sagalowsky A, Dawidson I, GlennieJ . Confirmation of the utility of fineneedleaspiration biopsy of the renal allograft. Kidney Int 1988; 34: 376-81.

23. Dantas M, Costa RS, Ferraz AS. Monitorização do al oenxerto renal através da biópsia aspirativa por agulha fina: relato de 218 punções realizadas na UTR do HCFMRP-USP. XVI Congresso Brasileiro de Nefrologia [Resumos]. 1992; 43.

24. Moura LAR, Alberti VN, Montezzo LC et al. Brazilian experience with fine-needle aspiration cytology in kidney transplants. Transplant Proc 1988; 20: 617-8.

25. Gonçalves LF, Manfro RC, Rauber L, Prompt CA. Evaluation of fine-needleaspiration biopsy in the diagnosis of acuterenal transplant dysfunction. Transplant Proc 1992; 24: 3.081-82, 1992.
26. Reeve RS, Cooksey G, Wenhan PW, Bourne LD, Paterson AD, et al. A comparison of fine needle aspiration cytol ogy and trucut tissue biopsy in the diagnosis of acute renal allograft rejection. Nephron 1986; 42:68-71.

27. Boshkos C, Steinmuller DR, Novick AC, Streem RJ , Cunningham RJ . Correlation of fine-needle aspiration biopsies with core biopsies after renal transplantation. Transplant Proc 1988; 22: 592-4.

28. Gupta R, Campbell J , Om A, Belitsky P. Serial monitoring of celular rejection by simultaneous histology and fine needle aspiration cytology. Transplant Proc 1985; 17: 2.123-4.

29. Hughes DA, McWhinnie DL, Sutton R, Chapman J R, Carter NP. Can incremental scoring of fine-needle aspirates predict histopathologic renal allograft rejection? Transplant Proc 1988; 20: 690-1.

30. Bolton EM, Thompson J F, Wood RF, Morris PJ . Immunoperoxidase staining of fine needle aspiration biopsies and needle core biopsies from renal allografts. Transplantation 1983; 36: 728-31.

31. Gonçalves LF, Rauber ML, Manfro RC, Prompt CA. Fine needle aspiration biopsy in renal transplant patients on prophylatic OKT3 treatment. Transplant Proc 1992; 24: 3.083-84.

32. J orgensen KA, Strate $M$, Svendsen $V$ et al. Fine needle aspiration biopsy during and after OKT3. Transplant Proc 1989; 21: 3.594-5.

33. Fletcher RH, Fletcher SW, Wagner EH. Diagnóstico. In Fletcher RH, Fletcher SW, Wagner EH (eds). E pidemiologia clínica. Porto Alegre, Artes Médicas, 1989; 68-107.

34. Castro MCR, Davi DSR, Saldanha LB et al. Should we treat a severe acute cellular rejection with no clinical signs? Transplantation 1991; 51:1.301-2.

35. Solez K, McGraw DJ , Beschorner WE et al. Reflections on the use of therenal biopsy as the "gold-standart" in distinguishing transplant rejection from cycl osporine nephrotoxicity. Transplant Proc 1985; 17: 123-33.

36. Egidi F, Banfi G, Bogetic J , Passerini P, Ponticelli C. Correlation between fine-needle aspiration biopsy and renal biopsy in renal transplantation. Transplant Proc 1988; 20: 589-91.

37. Belitsky P, Gupta R. Fineneedleaspiration biopsy. In Burdick J , Racusen LC, Solez K, Williams GM (eds). Kidney transplant rejection. $2^{\text {nd }}$ ed. New York, Marcel Dekker Inc, 1992; 419-35.

38. Harfmann P, Dittmer R, Busch R et al. Morphologic changes in the fine needle aspiration cytology of renal transplants during virus infection as detected by DNA in situ hybridization. Transplant Proc 1989; 21: 3.588-90.

39. Nguyen $L$, Hammer $C$, Dendorfer $U$ et al. Changes in large granular lymphocytes size and number in kidney transplant patients during rejection and viral infection. Transplant Proc 1985; 17: 2.110-1.

40. van Oers MHJ , Surachno S, Wilmink J M. Infiltrate analysis by monoclonal antibodies does not contribute to the usefulness of fineneedleaspiration biopsy. Transplant Proc 1987; 19: 1.646-9.

41. Belitsky P, Miller SM, Gupta R, Lee S, Ghose T. Induction of $\mathrm{MHC}$ class II expression in recipient tissuecaused by al lograft rejection. Transplantation 1990; 49: 472-6.

42. Von Willebrand E, Loginov R, Salmela K, I soniemi H, Häyry $P$. Relationship between intercellular adhesion molecule-1 and HLA class II expression in acute cellular rejection of human kidney allografts. Transplant Proc 1993; 25: 870-1.

43. Manfro RC, Gonçalves LFS, Rauber M, Moura LAR. Analysis of ICAM-1 and HLA-DR expression on renal allograft aspirates. Clin Transplant 1996; 10: 379. 